\title{
The Distribution and Ages of Star Clusters in the Small Magellanic Cloud: Constraints on the Interaction History of the Magellanic Clouds
}

\author{
Theodoros Bitsakis ${ }^{1}$ (D), R. A. González-Lópezlira ${ }^{1}$, P. Bonfini ${ }^{1}$, G. Bruzual ${ }^{1}$ (iD, G. Maravelias ${ }^{2,3}$, D. Zaritsky ${ }^{4}$ (D), \\ S. Charlot ${ }^{5}$ (D), and V. H. Ramírez-Siordia ${ }^{1}$ \\ ${ }^{1}$ Instituto de Radioastronomía y Astrofísica, Universidad Nacional Autónoma de México, Morelia, 58089, Mexico; t.bitsakis@irya.unam.mx \\ ${ }^{2}$ Instituto de Física y Astronomía, Universidad de Valparaíso, Valparaíso, Chile \\ ${ }^{3}$ Department of Physics, University of Crete, GR-71003 Heraklion, Greece \\ ${ }^{4}$ Steward Observatory, University of Arizona, Tucson, AZ 85719, USA \\ ${ }^{5}$ Sorbonne Universités, UPMC-CNRS, UMR7095, Institut d'Astrophysique de Paris, F-75014 Paris, France \\ Received 2017 October 10; revised 2017 December 12; accepted 2017 December 13; published 2018 January 26
}

\begin{abstract}
We present a new study of the spatial distribution and ages of the star clusters in the Small Magellanic Cloud (SMC). To detect and estimate the ages of the star clusters we rely on the new fully automated method developed by Bitsakis et al. Our code detects 1319 star clusters in the central $18 \mathrm{deg}^{2}$ of the SMC we surveyed (1108 of which have never been reported before). The age distribution of those clusters suggests enhanced cluster formation around $240 \mathrm{Myr}$ ago. It also implies significant differences in the cluster distribution of the bar with respect to the rest of the galaxy, with the younger clusters being predominantly located in the bar. Having used the same setup, and data from the same surveys as for our previous study of the LMC, we are able to robustly compare the cluster properties between the two galaxies. Our results suggest that the bulk of the clusters in both galaxies were formed approximately $300 \mathrm{Myr}$ ago, probably during a direct collision between the two galaxies. On the other hand, the locations of the young ( $\leqslant 50 \mathrm{Myr}$ ) clusters in both Magellanic Clouds, found where their bars join the H I arms, suggest that cluster formation in those regions is a result of internal dynamical processes. Finally, we discuss the potential causes of the apparent outside-in quenching of cluster formation that we observe in the SMC. Our findings are consistent with an evolutionary scheme where the interactions between the Magellanic Clouds constitute the major mechanism driving their overall evolution.
\end{abstract}

Key words: catalogs - galaxies: star clusters: general - Magellanic Clouds - methods: statistical

Supporting material: machine-readable table

\section{Introduction}

The Magellanic Clouds have significantly advanced our understanding on galaxy evolution. Owing to their proximity, individual stars can be observed, providing important information about the spatially resolved star formation and the origin and properties of their stellar populations.

The Small Magellanic Cloud (SMC) is a dwarf irregular galaxy located at a distance of $\sim 60.6 \mathrm{kpc}$ (Hilditch et al. 2005). Simulations supported by observational evidence suggest that it evolved in tandem with its counterpart-the Large Magellanic Cloud (LMC), thus sharing a common interaction and star formation history (e.g., see Besla et al. 2012 and references therein). Yoshizawa \& Noguchi (2003) performed $N$-body simulations of the tidal distortions and concluded that the two galaxies should have interacted over the past $\sim 0.2 \mathrm{Gyr}$. Their results are partially supported by Harris \& Zaritsky (2004), who studied the spatially resolved star formation history of the SMC and showed that it underwent various periods of enhanced star formation $\sim 2.5,0.4$, and $0.06 \mathrm{Gyr}$ ago. They are also in agreement with Chiosi et al. (2006) and Glatt et al. (2010), who suggested that the close interaction between the two Clouds have resulted in the triggering of cluster formation activity.

More recently, Besla et al. (2007) and Kallivayalil et al. (2013) challenged the scenarios where the Magellanic Clouds have already completed several orbits around the Galaxy, using current Hubble Space Telescope (HST) proper motion measurements; they suggested that the Clouds are in their first orbit passage about the Galaxy. Moreover, Besla et al. (2012) studied the interaction history of those galaxies using numerical models constrained by the HST observations and showed that, while they have not interacted before with the Galaxy, the Magellanic Clouds must have experienced a direct collision some time 100-300 Myr ago. This seems to agree with the findings of Harris (2007), who studied the stellar populations of the Magellanic Bridge - the tidal stream of neutral gas and stars possibly associated with the interaction of the two galaxiesand showed that the star formation in the Bridge commenced some time 200-300 Myr ago. A direct cloud-cloud collision would also explain the existence of a small population of SMC stars-based on their peculiar kinematics and metallicitieswhich were found in the LMC (Olsen et al. 2011). In spite of all this progress, the question of whether the evolution of the Magellanic Clouds is driven by internal processes (i.e., the action of bars, morphological/dynamical quenching) or environmental mechanisms (i.e., galaxy interactions) is still unclear. One would expect that in the case of environmental evolution many of the properties of the two galaxies (e.g., the star formation history) would be correlated.

A robust method to explore the formation and interaction histories of nearby galaxies, where individual stars can be resolved (such as the Magellanic Clouds), entails the study of the age distribution of their star clusters. Owing to modern instrumentation that allows us to estimate their ages and metallicities with high precision-in contrast with field starsstar clusters represent unique tools to constrain the star formation history of their host galaxies and to disentangle the 

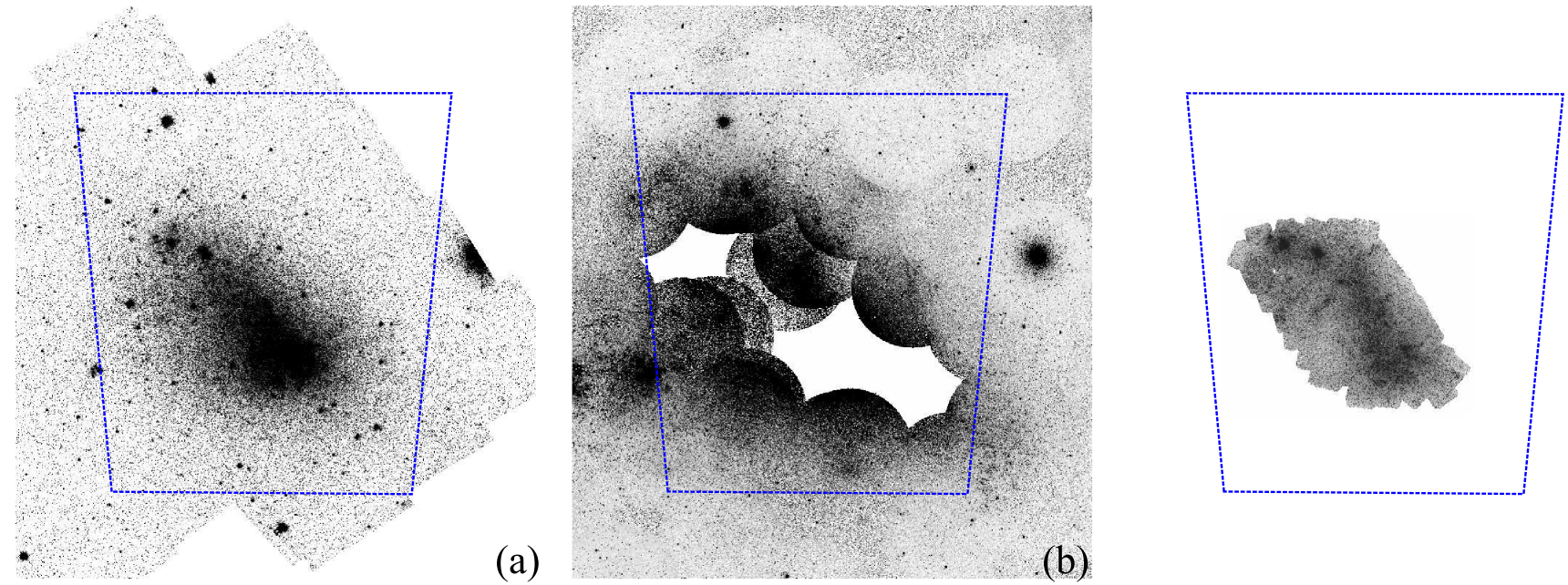

Figure 1. (a) The Spitzer/IRAC $3.6 \mu \mathrm{m}$ (Gordon et al. 2011), (b) the GALEX/NUV (Simons et al. 2014), and (c) the Swift/UVOT (Siegel et al. 2014) mosaics of the SMC, respectively. The dashed blue box indicates the area covered by MCPS (Zaritsky et al. 2002), which was also surveyed by our code.

special conditions they might have undergone. Despite the plethora of studies of the star clusters in the Magellanic Clouds, the lack of a statistically robust detection method that creates uniform and complete samples (as opposed to the visual identification methods that are usually applied) has posed significant limitations for the systematic study of the star cluster formation history of both galaxies. In Bitsakis et al. (2017), we presented a new fully automated method to robustly detect and estimate the ages of star clusters in nearby galaxies. Using statistical analysis on high-resolution maps of the LMC, we obtained a large, uniform sample of star clusters (in the central $49 \mathrm{deg}^{2}$ ), which we exploited to put constraints on the formation history of that galaxy. A similar analysis is followed in the current study using the same method and data surveys for the SMC. In Section 2, we describe the data set we use in the current study. Section 3 contains a brief description of the cluster detection and age estimation codes (a more analytic description along with statistical tests can be found in Bitsakis et al. 2017). The results are presented in Section 4, while in Section 5 we make a comparison of the SMC-LMC star cluster age distributions and derive useful conclusions about their interaction history. Finally, in Section 6, we summarize our findings.

Throughout this work, we assume a distance modulus to the SMC of 18.91 mag (Hilditch et al. 2005).

\section{The Data}

We have made use of archival data of the SMC at various bands. Simons et al. (2014) presented the near-ultraviolet mosaic ( $\lambda_{\text {eff }}=2275 \AA$ ) of that galaxy obtained by the Galaxy Evolution Explorer (GALEX; Martin et al. 2005). The median exposure time was $733 \mathrm{~s}$, and the $5 \sigma$ depth of point sources varied between 20.8 and $22.7 \mathrm{mag}$. Although the mosaic covers a region of $63 \mathrm{deg}^{2}$, which contains the SMC bar, wing and tail, there are two sub-regions that were not observed, of $\sim 0.25$ and 1 deg diameter, northeast and southwest from the center, respectively (see Figure 1(b)). These holes in the coverage were compensated for with the Swift Ultraviolet-Optical Telescope (UVOT) Magellanic Clouds Survey (SUMAC; Siegel et al. 2014), which imaged the central $3.8 \mathrm{deg}^{2}$ of the galaxy (Figure 1(c)) with deeper exposures of $3000 \mathrm{~s}$ in all three $N U V$ filters of the instrument $(U V W 1$, $U V W 2$, and $U V M 2$ ).
Our infrared data come from the "Surveying the Agents of a Galaxy's Evolution SMC survey" (SAGE-SMC; Gordon et al. 2011) that mapped the full SMC $\left(30 \mathrm{deg}^{2}\right)$ with both the Infrared Array Camera (IRAC; Figure 1(a); Fazio et al. 2004) and the Multiband Imaging Photometer (MIPS; Rieke et al. 2004) on board the Spitzer Space Telescope. It produced mosaics at 3.6, 4.5, 5.8, and $8.0 \mu \mathrm{m}$ with IRAC and at 24, 70, and $160 \mu \mathrm{m}$ with MIPS, with integrated exposure times of $63 \mathrm{hr}$ in the IRAC and $\sim 400 \mathrm{hr}$ in the MIPS bands, respectively.

Finally, we exploited the photometric information by Zaritsky et al. (2002), who presented the stellar catalog and extinction map of the SMC, as part of the Magellanic Cloud Photometric Survey (MCPS; marked with dashed blue lines in Figure 1). They obtained 3.8-5.2 minute exposures of the central $18 \mathrm{deg}^{2}$ of the SMC in the Johnson $U, B, V$, and Gunn $i$ bands with the Las Campanas Swope Telescope under 1.5 arcsecond seeing conditions. The limiting magnitudes varied, depending on the filter, between $21.5 \mathrm{mag}$ for $U$ and $23.0 \mathrm{mag}$ for $i$. Using DAOPHOT II (Stetson 1987), they created a photometric catalog that contains 24.5 million sources in all the area covered by the MCPS (including the SMC, LMC, and the Magellanic Bridge). They also estimated the line-of-sight extinctions to the stars in their catalog and produced an extinction map of the SMC. This was achieved by comparing the observed stellar colors with those derived from the stellar photospheric models of Lejeune et al. (1997). Thus, they estimated the effective temperature $\left(T_{\text {eff }}\right)$ and measured the extinction $\left(A_{V}\right)$ along the line of sight to each star, adopting a standard Galactic extinction curve. They produced two $A_{V}$ maps, one for hot $\left(12,000 \mathrm{~K}<T_{\text {eff }} \leqslant 45,000 \mathrm{~K}\right)$ and one for cool $\left(5500 \mathrm{~K}<T_{\text {eff }} \leqslant 6500 \mathrm{~K}\right)$ stars. In Figure 1, we present the coverage of MCPS in comparison with that of other surveys we used for the detection of the star clusters; one can see that the central $18 \mathrm{deg}^{2}$ of the SMC are imaged.

\section{The Cluster Detection and Age Estimation Method}

The code we used here to automatically detect and estimate the ages of the SMC star clusters was analytically described in Bitsakis et al. (2017). Summarizing, the code makes use of the star counts method (see Schmeja 2011 and references therein), which estimates the density of stars in a given region of interest and finds overdensities above some local background thresholds 
Table 1

SMC Star Cluster Catalog

\begin{tabular}{|c|c|c|c|c|c|c|c|}
\hline Catalog ID & $\begin{array}{l}\text { R.A.(J2000) } \\
\quad(\mathrm{deg})\end{array}$ & $\begin{array}{l}\text { Decl.(J2000) } \\
\quad \text { (deg) }\end{array}$ & $\begin{array}{c}\text { Radius } \\
(\text { deg) }\end{array}$ & $\begin{array}{c}\log (\text { Age }) \\
\text { (years) }\end{array}$ & $\begin{array}{l}\text { Lower unc. } \\
\text { (years) }\end{array}$ & $\begin{array}{l}\text { Upper unc. } \\
\text { (years) }\end{array}$ & $\begin{array}{c}\text { Bica et al. (2008) } \\
\text { Catalog ID }\end{array}$ \\
\hline SMC-NUV-484 & 14.0765 & -72.4634 & 0.0280 & 7.22 & 6.92 & 7.33 & 343 \\
\hline SMC-M2-287 & 13.0482 & -72.5310 & 0.0065 & 7.99 & 7.77 & 8.01 & 258 \\
\hline
\end{tabular}

Note. The lower and upper uncertainty bounds are estimated at the 16th and 84th percentiles, respectively.

(This table is available in its entirety in machine-readable form.)

$\left(\Sigma_{\text {det }}\right)$. To define the relation between $\Sigma_{\text {det }}$ and the background density, we performed Monte Carlo simulations with artificial star clusters, having both Gaussian as well as uniform overdensity profiles (accounting for both compact and diffuse clusters), projected over various background values. The code is applied on a pixel-map conversion of the original image, where each star is represented by a single pixel. Only stars located in the overdensities are considered and a source detection is applied on the smoothed final image to define the center and radius of each candidate cluster. The method has been proven to be fast and accurate and was initially tested on the LMC with impressive results (see Bitsakis et al. 2017), yielding the discovery of 3500 new star clusters that have never been reported before. For the sake of consistency we use the same setup as for the LMC; we run the detection sequence on the ultraviolet (GALEX/NUV, Swift/UVM2) and near-infrared mosaics (Spitzer/IRAC 3.6) of the SMC in order to probe different cluster ages (e.g., young clusters are expected to host massive UV-emitting stars, while old clusters are dominated by low-mass stars emitting mostly in the near-IR part of the spectrum). We then use the MCPS catalog to obtain the photometric information of the stellar populations. The detection sequence yields a total of 2219 candidate clusters and associations in the corresponding region.

The age estimation algorithm (also presented in Bitsakis et al. 2017) consists of a modified version of the code of V. H. Ramírez-Siordia et al. (2017, in preparation). Briefly, this code uses a Bayesian approach to obtain the most likely theoretical isochrone that reproduces the observed CMD of each candidate cluster, while taking into account the cluster star memberships. The set of 80 model isochrones we used here is a byproduct of an independent project by S. Charlot \& G. Bruzual (2017, in preparation) ${ }^{6}$ and was produced following the evolutionary tracks of Chen et al. (2015) and accounting for the evolution of thermally pulsing asymptotic giant branch (TP-AGB) stars (Marigo et al. 2013). The isochrones were calculated for a representative SMC metallicity of $[\mathrm{Fe} / \mathrm{H}]=$ -0.70 (i.e., $Z=0.004$; Venn 1999) and cover the range $6.9 \leqslant \log ($ age $)<9.7$ years.

As anticipated above, we also perform field star decontamination. Our code uses a modified version of the method described in Mighell et al. (1996). According to this, the code produces the CMD of the candidate cluster as well of its surrounding field stars and estimates the probability of each candidate star to belong to the cluster. This membership probability is stored in a table containing all of the cluster star information and is eventually used during the age estimation process mentioned above. In Bitsakis et al. (2017), we showed

\footnotetext{
6 The Charlot \& Bruzual isochrones are available to the interested user upon request.
}

that the method performs well even in high field star density environments (such as the LMC/SMC bar). Eventually, the code discards any candidate cluster with an insignificant number of stars $(n<20)$ having high membership probability $(>60 \%)$, as well as those clusters that could not be fitted by our age estimation code.

To ensure a more accurate age estimation, we perform the CMD fitting in the $(U-V)$ versus $V,(B-V)$ versus $V$, and $(V-i)$ versus $i$ bands for each cluster, and then we combine the final results using Equation (5) from Bitsakis et al. (2017), which takes into account the number of stars included and how well the age is constrained in each fitting. In Figure 2, we present two examples of the best age estimation in the CMDs of clusters SMC-NUV-484 and SMC-IR1-727. The final catalog contains 1319 secure clusters (40\% smaller than the initial candidate cluster sample). These clusters are presented in Table 1; column (1) gives the cluster identifier (it consists of a reference to the band where each cluster was initially detected, i.e., $I R 1$ refers to Spitzer/IRAC1, NUV to GALEX/NUV, and $M 2$ to Swift/UVM2, plus the serial number of the corresponding cluster); columns (2) and (3), respectively, contain the right ascension (R.A.) and declination (decl.) of the cluster centers, in J2000 decimal equatorial coordinates; column (4) reports the cluster radii; columns (5), (6), and (7) contain, respectively, the best age estimation for each cluster, and its lower and upper uncertainty bounds (derived from the 16th and 84th percentiles of the probability distribution histogram produced by the code); finally, column (8) contains - if available - the corresponding cluster identifier from the catalog of Bica et al. (2008). Some characteristic examples of clusters ordered by increasing age are presented in Figure 3.

\section{Results \\ 4.1. Comparisons with Other Surveys}

We compare our final catalog of star clusters with that of Bica et al. (2008). These authors have reported 515 clusters in the central $18 \mathrm{deg}^{2}$ of the SMC we surveyed, 211 of which $(58 \%)$ overlap our sample. In Figure 4, we compare our age estimates with those from other surveys. Rafelski \& Zaritsky (2005) compared the integrated colors of their star clusters, acquired from the MCPS survey, with models of simple stellar populations. Unfortunately, their technique is not able to decontaminate from field stars; hence, although these authors performed various tests to ensure the reliability of their estimates, their method can introduce significant biases, especially at high field star density regions (like the SMC bar). Thus, the comparison with their results yields a Pearson $R$-coefficient 0.74 (see also Figure 4(a)). On the other hand, Glatt et al. (2010) visually fitted a set of isochrone models to 

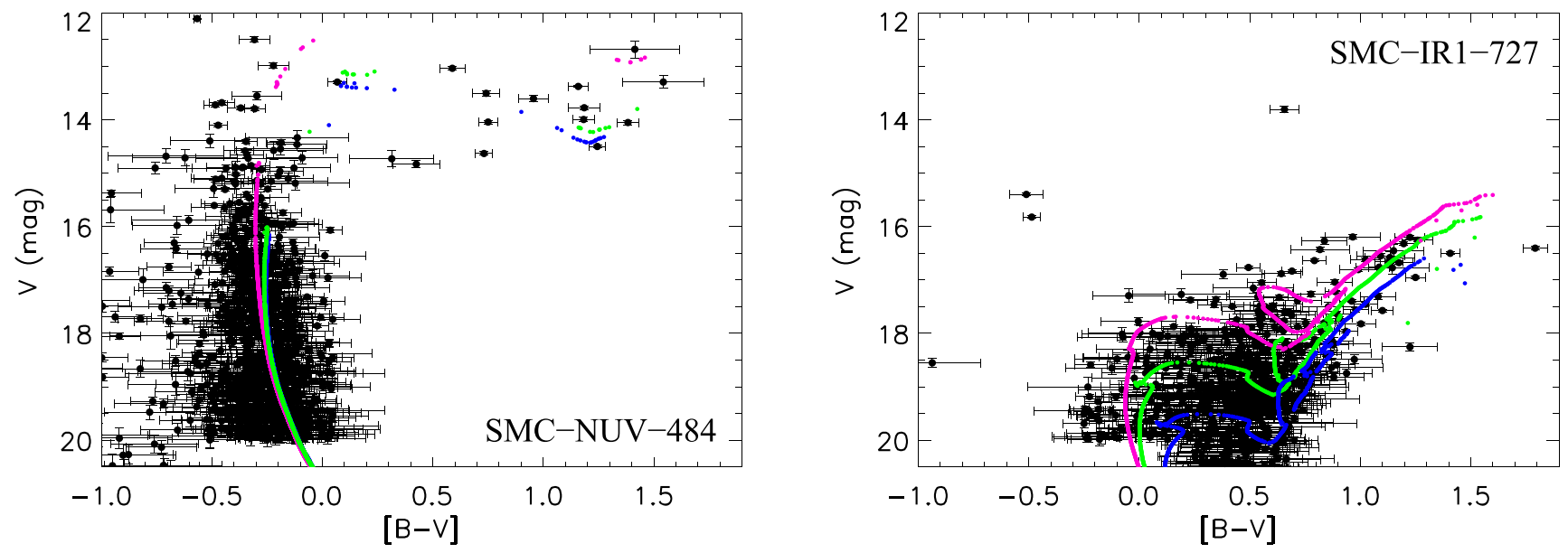

Figure 2. Examples of the isochrone fitting process in the $(B-V)$ vs. $V$ field star decontaminated CMDs of the star clusters SMC-NUV-484 and SMC-IR1-727, presented in Figure 2. Best-fit isochrones are presented in green, and upper and lower uncertainties in magenta and blue, respectively.

the observed cluster CMDs. Although they used a field star decontamination technique, the large uncertainties introduced by visual identification of the main-sequence turnoff are likely the origin of the large scatter between theirs and our age estimates, having $R=0.82$ (see Figure 4(b)). Similarly, Chiosi et al. (2006) corrected for field star contamination, and used both visual and $\chi^{2}$ minimization methods; they divided the observed and model CMDs in bins of color and magnitude and minimized their differences. Although we only have 11 clusters in common, the comparison yields $R=0.77$ (see Figure 4(c)). Finally, Parisi et al. (2014) carefully calculated the ages of a small sample of 15 old SMC clusters using high spatial resolution data from the Very Large Telescope in Chile. For the only cluster we have in common (identified as L17 in their catalog, our SMC-IR1-226), we measure an age 1.22 $-0.40 \mathrm{Gyr}$, which is remarkably similar to their 1.25 Gyr estimate.

\subsection{The Age Distribution of Star Clusters}

In Figure 5, we present the age distribution of star clusters in the SMC. The bin size was optimized using the FreedmanDiaconis rule (bin size $0.136 \mathrm{dex}$ ). The main cluster formation event seems to have happened $\sim 240 \mathrm{Myr}$ ago. The decline in the number of star clusters beyond the main peak could be associated both with cluster fading (e.g., Boutloukos \& Lamers 2003) and/or cluster dissolution due to a variety of mechanisms, such as (i) residual gas expulsion, (ii) two-body relaxation, (iii) tidal heating from disk shocks, and (iv) tidal harassment from giant molecular clouds (see Baumgardt et al. 2013 and references therein). On the other hand, phenomena like the cluster disruption due to gas expulsion after the burst of star formation took place in the initial stages of cluster formation, and therefore in short timescales ( $\sim 40 \mathrm{Myr}$ for the Magellanic Clouds; see de Grijs \& Goodwin 2009).

Since a star cluster formation event in our data could be represented by a single Gaussian distribution (due to the range of uncertainties in the estimation of the cluster ages), we use a Gaussian mixture model code, NMIX, ${ }^{7}$ to derive the underlying number of such distributions in our data. This method reports the statistically motivated number of Gaussian distributions that

Publicly available at https://people.maths.bris.ac.uk/ mapjg/Nmix. can fit a given data set by implementing the approach of Richardson \& Green (1997). In Figure 6, we present the results of the fitting; it is shown that our cluster age distribution can be successfully reproduced by a three-component mixture model (having Bayes $K$-factors between that model and each one of the rejected univariate distributions $>4.5$ ), with peaks 30, 240, and $680 \mathrm{Myr}$ ago. Based on their results, Glatt et al. (2010) have visually identified and proposed two main periods of cluster formation 160 and $630 \mathrm{Myr}$ ago, as well as a minor event $\sim 50$ Myr ago (see Figure 5 of that work); this last event of star formation was also detected by Harris \& Zaritsky (2004). Whereas the 50 and $630 \mathrm{Myr}$ peaks from Glatt et al. (2010) are consistent with our secondary cluster formation events, the $160 \mathrm{Myr}$ one is significantly different from our main $240 \mathrm{Myr}$ event. We note here that histogram peaks also can be the result of binning artifacts. This is not the case for our findings since NMIX fits models on the unbinned data. To test whether binning could be at the origin of the discrepancy with Glatt et al. (2010), we applied the Freedman-Diaconis rule to calculate the bin size for their sample; its value is 0.109 dex. Using this bin size, we produced an updated version of the Glatt et al. (2010) histogram, which shows a major formation event $280 \mathrm{Myr}$ ago, with minor ones appearing 20, 100, and $450 \mathrm{Myr}$ ago. This exercise suggests that, in addition to the scatter mentioned in Section 4.1, differences in the binning scheme also contribute to the different results obtained by Glatt et al. (2010) and in the present work.

\subsection{The Spatial Age Distribution of Star Clusters}

To further study the cluster formation history in the SMC, we present in the two panels of Figure 7 the age distributions of those clusters located in the bar (left panel) and everywhere else in the galaxy (hereafter referred to as " "outskirts"; right panel). The two distributions display important differences, having a Kolmogorov-Smirnov probability of being drawn from the same sample $<10^{-5}$. In contrast to the bar that had a major formation event around $200 \mathrm{Myr}$ ago, with secondary peaks appearing at 20 and $\sim 800 \mathrm{Myr}$, the "outskirts" major peak appeared 270 Myr ago, with secondary ones $40 \mathrm{Myr}$ and $2 \mathrm{Gyr}$ ago. These results are drawn from the three-component NMIX models, having $K$-factors $>3.9$ (see Figure 7). Although the two major peaks might be associated with the same cluster formation event, it is possible that 



\section{R.A. (J2000)}

Figure 3. Examples of clusters from our catalog presented on the Spitzer IRAC $3.6 \mu \mathrm{m}$ image. The dashed black lines mark the radii, as defined by the star-count code. Clusters (a) SMC-NUV-484, age 16.6-4.7 Myr; (b) SMC-IR1-665, age 48.5 ${ }_{-2.0}^{+2.9}$ Myr; (c) SMC-IR1-635, age 186-35 Myr; (d) SMC-IR1-358, age 512 $2_{-124}^{+135}$ Myr; (e) SMCIR1-727, age $845_{-650}^{+284} \mathrm{Myr}$; and (f) SMC-IR1-270, age 1.07-0.85 Gyr. The horizontal and vertical axes correspond to R.A. and decl. measured in degrees (J2000).

the bar delayed its cluster formation with respect to the rest of the galaxy. Furthermore, the skewness of the outskirts distribution suggests a sudden termination of the cluster formation, contrary to the more continuous formation in the bar.

The above results can be also confirmed from Figure 8, where we present the spatial distribution of clusters of different ages in our sample (the age ranges are as in Bitsakis et al. 2017). Clusters younger than $100 \mathrm{Myr}$ are solely located in the bar region, while clusters older than $355 \mathrm{Myr}$ are mostly populating the outskirts. The bar is also associated with two prominent H I supershells (Stanimirovic et al. 1999), confirming the recent burst of star formation in that region. What is remarkable is the fact that, starting from the center of the SMC bar, clusters of larger ages are gradually located outward, with only very few old clusters (>750 Myr) found in the central region of the galaxy. This result suggests that an outside-in quenching of cluster formation occurred over the past Gyr in the SMC.

\section{Discussion: Comparison between the LMC-SMC Cluster Ages and Implications}

As presented above, our method is able to create complete, uniform samples of star clusters that allow comparisons between different galaxies. In particular, the use of an identical setup and data as in Bitsakis et al. (2017) secures the robustness 

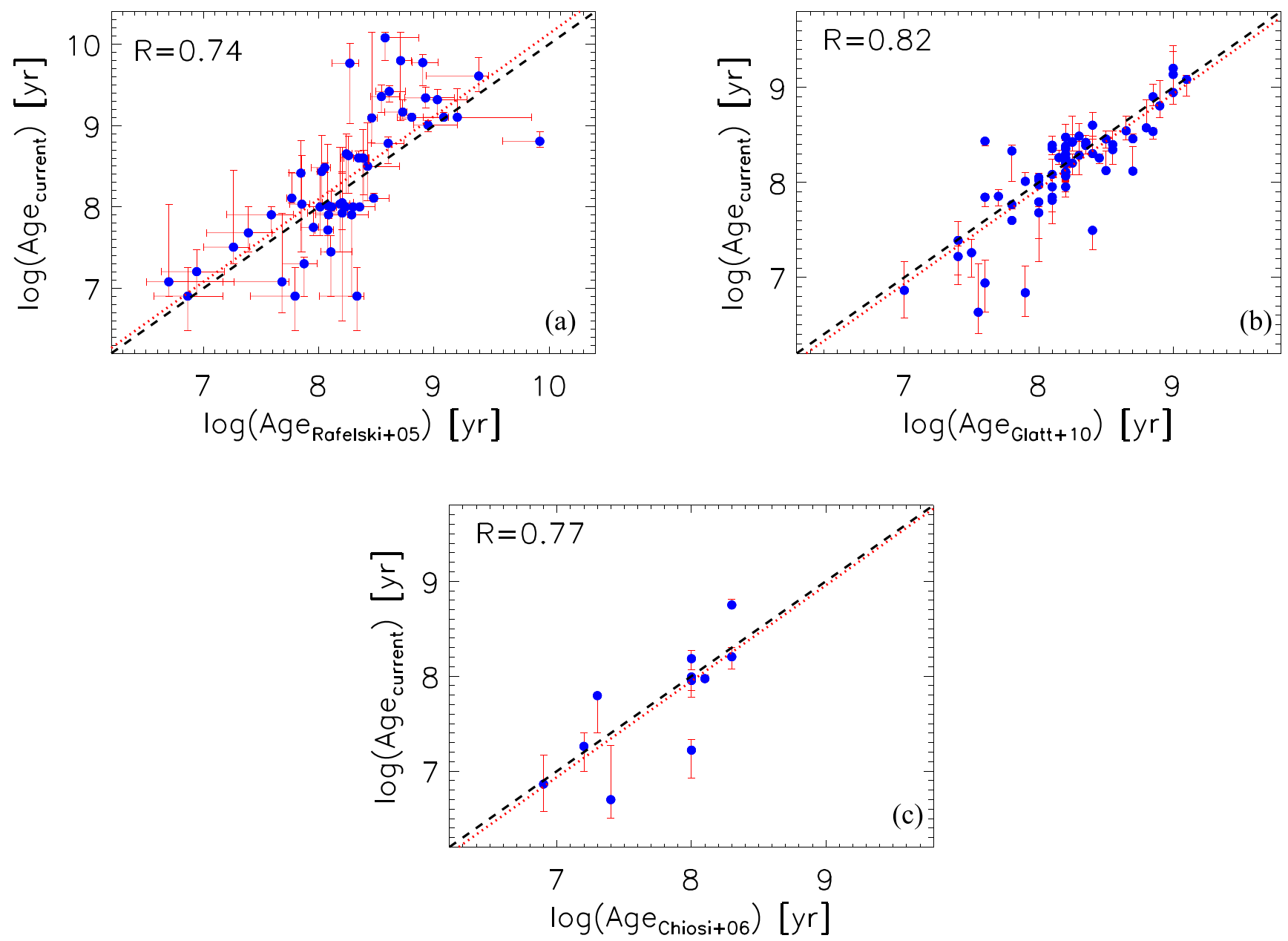

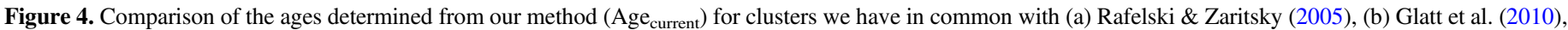
and (c) Chiosi et al. (2006). The dashed black lines correspond to the one-to-one correlation, while the dotted red ones are the least squares fits to the data. The Pearson correlation coefficients $(\mathrm{R})$ are indicated in the upper left corner of each panel.

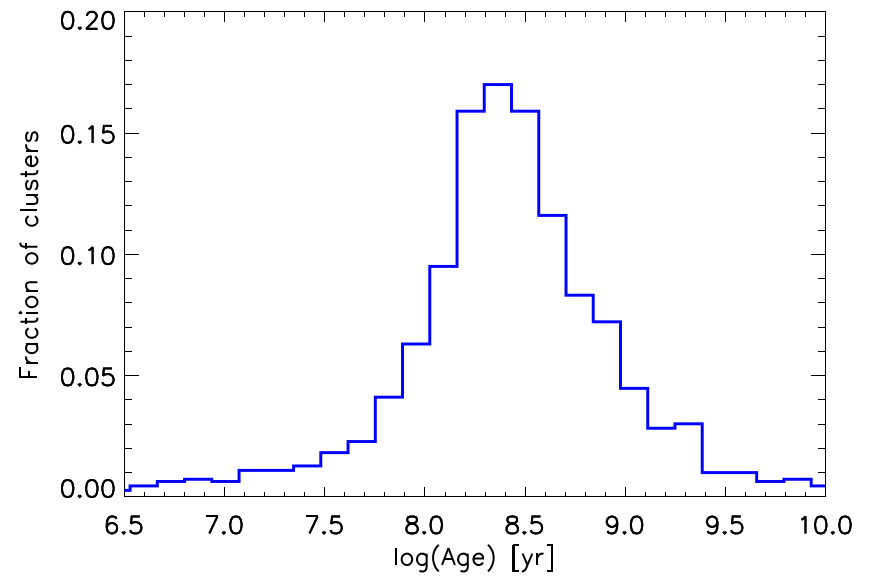

Figure 5. Age distribution of the SMC clusters. The fractions presented here are normalized to the total number of clusters found in that galaxy.

of the comparisons between the star cluster properties of the two Magellanic Clouds, namely, the SMC and LMC.

We compare the cluster age distributions of the two galaxies presented in Figure 5 of the current work for the SMC and in Figure 8 of Bitsakis et al. (2017) for the LMC, and we discuss the implications. The comparison shows that both Clouds display enhanced cluster formation activity in the past

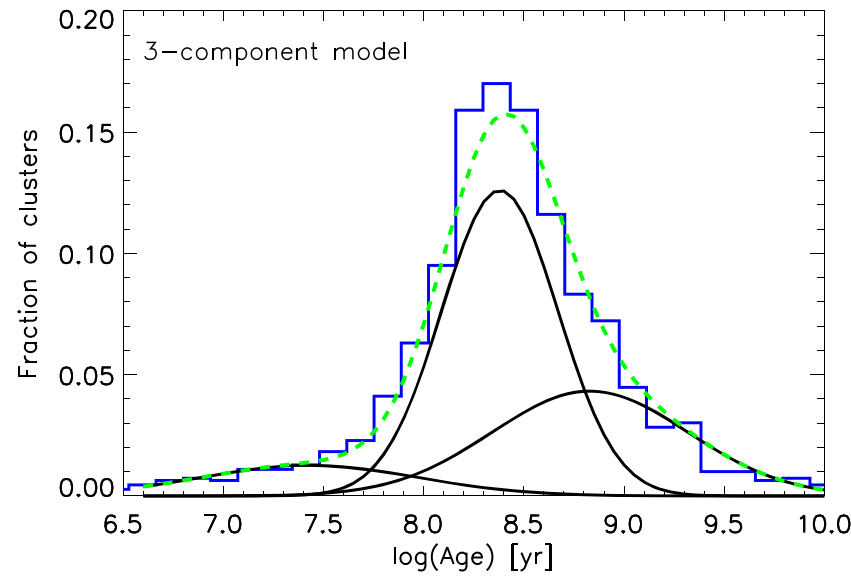

Figure 6. Three-component mixture model (dashed green line), and its individual constituents (solid black lines). The fractions presented here are normalized to the total number of clusters found in that galaxy.

200-300 Myr. This is also consistent with the peaks of cluster formation in the bars of both galaxies; this age coincides with the epoch at which Besla et al. (2012) estimated that a direct collision occurred between the two Clouds. Yet, owing to large differences in their sizes and masses, the effects of such a collision in the cluster formation history of the two galaxies should have been very different. This is evident in Figure 9, 

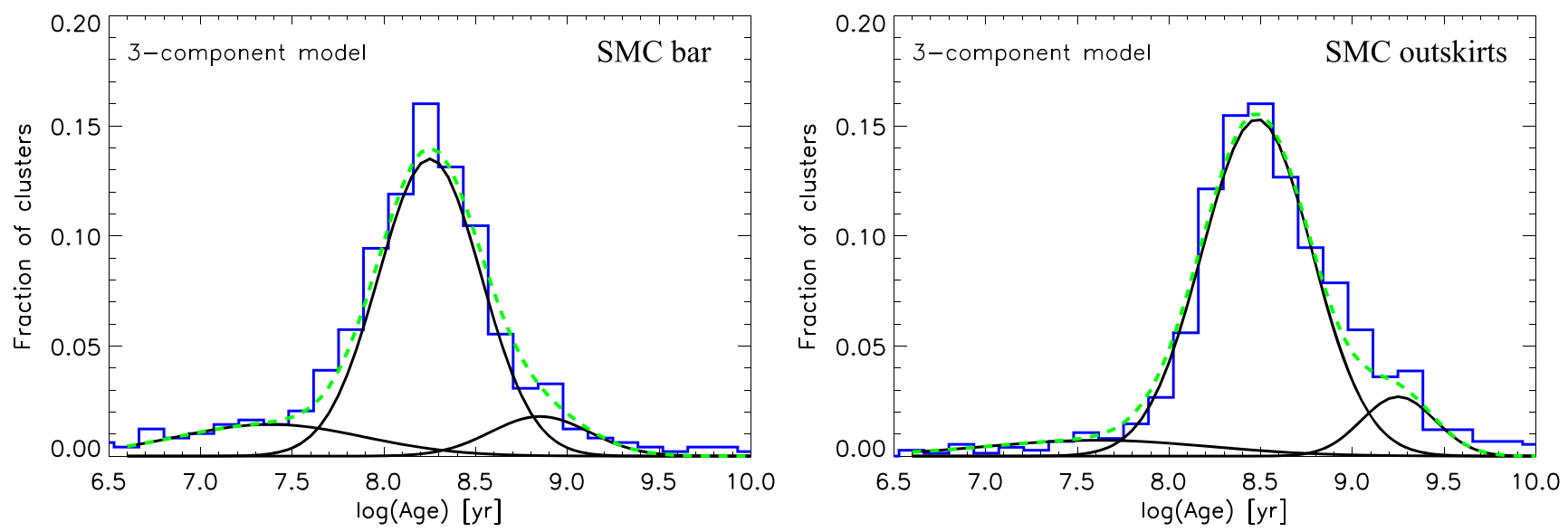

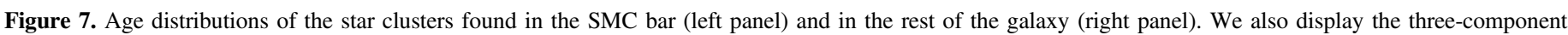

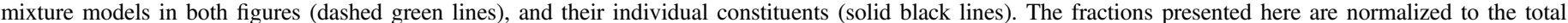
number of clusters found in that galaxy.

where we present the median age distribution in bins $\sim 0.5 \mathrm{deg}^{2}$ for the LMC (left) and the SMC (right), respectively. It is shown that the star clusters in the SMC bar are younger than those in the LMC bar, where the most recent cluster formation occurred >50 Myr ago. In contrast, the SMC bar is experiencing an ongoing cluster formation activity, with $8 \%$ of its clusters (14\% of those located in the bar) having ages $<50$ Myr. This also agrees with the findings of Chiosi et al. (2006) and Glatt et al. (2010) of very recent $(<20 \mathrm{Myr})$ cluster formation activity in the SMC. This suggests the presence of cold molecular gas in the central region of that galaxy, as confirmed by Bolatto et al. (2011).

The age distributions of the outskirts of both galaxies also show great differences. The SMC contains, on average, clusters older than $300 \mathrm{Myr}$ ( $\sim 15 \%$ of them are older than a Gyr), while the LMC contains mostly clusters $150-500$ Myr old (only $7 \%$ have ages $>1$ Gyr). Despite those differences, both distributions seem to have peaked $\sim 300 \mathrm{Myr}$ ago, suggesting that the aforementioned collision between the two Clouds not only affected their bars, but rather triggered cluster formation on a global scale in those galaxies. The secondary SMC peak at 680 Myr might be matched with the smaller $~ 500$ Myr peak of the LMC. These results would then be in agreement with the $0.6 \mathrm{Gyr}$ star formation enhancement observed by Harris \& Zaritsky (2004, 2009), who studied the star formation histories of the two galaxies and, based on orbital simulations available at the time, associated such events with perigalactic passages of the Magellanic Clouds about the Galaxy.

This difference in the old versus young cluster spatial distributions suggests that the SMC may have ceased its star cluster formation in an outside-in fashion. This result is consistent with the findings of Cignoni et al. (2013), who studied the spatially resolved star formation history of six SMC regions and suggested the existence of an age gradient with all the star formation activity over the past $0.5 \mathrm{Gyr}$ being concentrated in the central region. Such an age gradient has not been reported, however, for clusters older than $1 \mathrm{Gyr}$ (see Parisi et al. 2014). This implies that its interaction with the LMC (or the Galaxy) could have affected (by stripping, shocks, or inflows toward the center) its outer gas reservoir, thus preventing it from forming younger star clusters in the outskirts. Zhang et al. (2012) studied the multiband surface brightness profiles of 34 nearby dwarf irregular galaxies, and found an outside-in shrinking of the star formation that they attributed to environmental effects (i.e., interactions between galaxies). Arguably, the LMC, being 50\% more massive than the SMC, did not suffer similar gas loss by galaxy-galaxy interactions, and hence retained its global cluster formation throughout its lifetime.

The comparison of the spatial distributions of young clusters $(<50 \mathrm{Myr})$ in the Magellanic Clouds is also puzzling. As shown in Figure 9, clusters with these ages in the SMC are mostly located at the bar, preferentially at the bar-"arm" junction points, while in the LMC they lie mostly along its arms. In the case of the LMC, H I arms are found northeast and southwest of the bar (Kim et al. 2003), whereas in the SMC they trace an elongated structure located southeast of the bar (Stanimirovic et al. 1999; Dickey et al. 2000). Interestingly, Ochsendorf et al. (2017) showed that the most active starforming regions at present in the LMC, namely, the 30Dor and N79, are located where the LMC bar joins the H I arms. Such locations are very likely to enhance star formation due to the high concentrations of gas and to shocks induced by the internal dynamics, and very young stars/clusters have been observed there in various other galaxies (e.g., Beuther et al. 2017). The absence of young clusters in the outskirts of the SMC is likely due to the overall scarcity of gas in the last few Myr. The hypothesis of outside-in stripping of the gas in the SMC is also consistent with the cold molecular gas distribution (see Bolatto et al. 2011). In the SMC, molecular gas is mostly confined to the bar, and indeed its youngest clusters overlap the densest molecular gas in the northeastern portion of the bar at its intersection with the aforementioned H I feature. Using the Spitzer/MIPS $24 \mu \mathrm{m}$ images we confirm that the locations of the young clusters coincide with those of the warm dust clouds too. It is plausible that many of those clusters are still embedded in the progenitor clouds, thus explaining their very young ages.

Regarding the LMC, since many of the clusters younger than $50 \mathrm{Myr}$ seem to trace both H I arms (Bitsakis et al. 2017), we have considered the possibility that star formation there is related to a long-lived spiral density wave not connected to an interaction with the SMC. This hypothesis, however, is

\footnotetext{
8 The "arms" here are intended as those H I features of the Magellanic Clouds resembling classical spiral arms, although their actual nature is still under debate, as described in the text.
} 

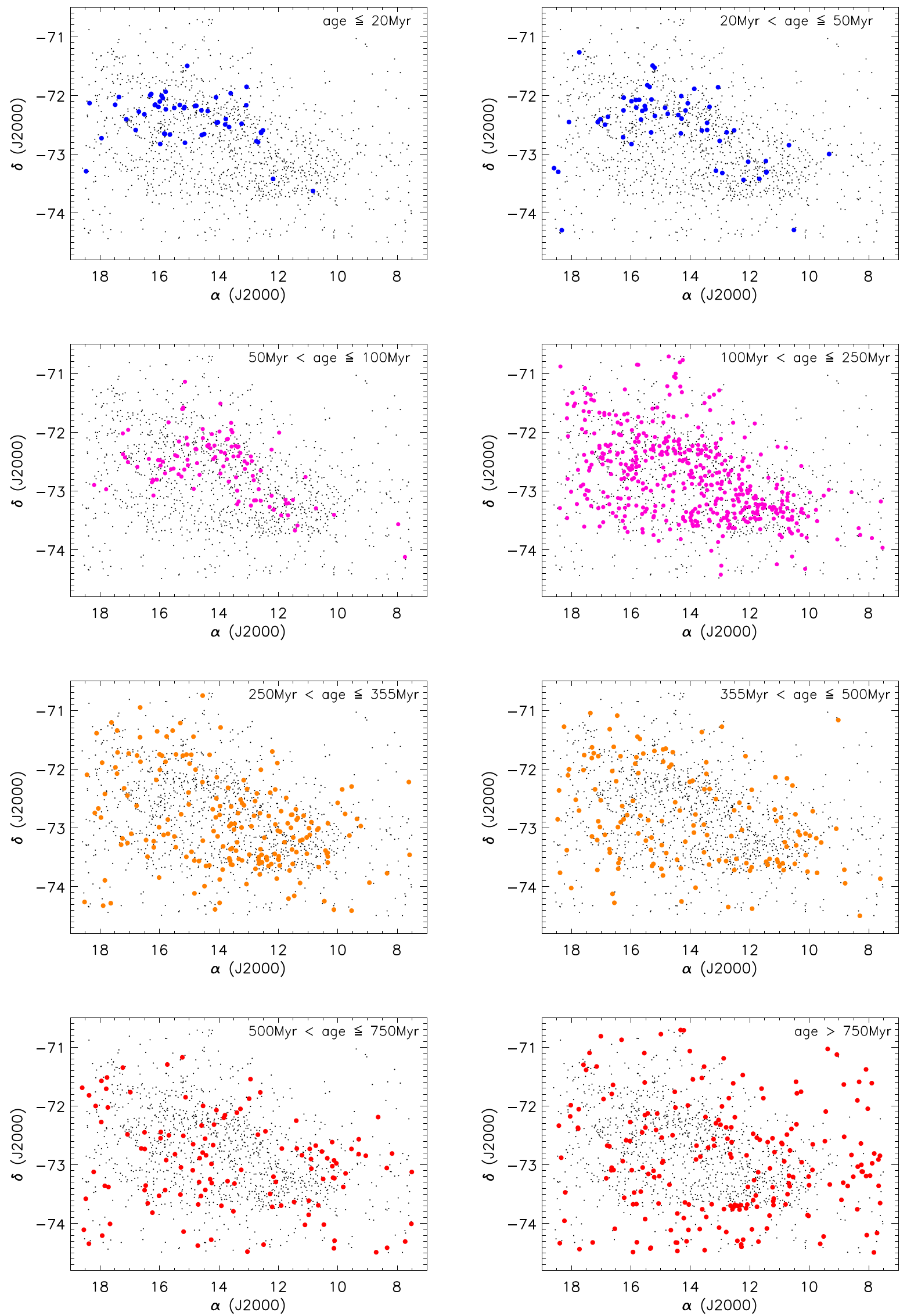

Figure 8. Spatial age distribution for all the star clusters in our sample (black dots). The coordinates in both axes are in degrees (J2000). From top left to bottom right, we present the positions of star clusters with: Age $\leqslant 20 \mathrm{Myr}, 20<$ Age $\leqslant 50 \mathrm{Myr}, 50<$ Age $\leqslant 100 \mathrm{Myr}, 100<$ Age $\leqslant 250 \mathrm{Myr}, 250<$ Age $\leqslant 355 \mathrm{Myr}$, $355<$ Age $\leqslant 500 \mathrm{Myr}, 500<$ Age $\leqslant 750 \mathrm{Myr}$, and Age $>750 \mathrm{Myr}$. 

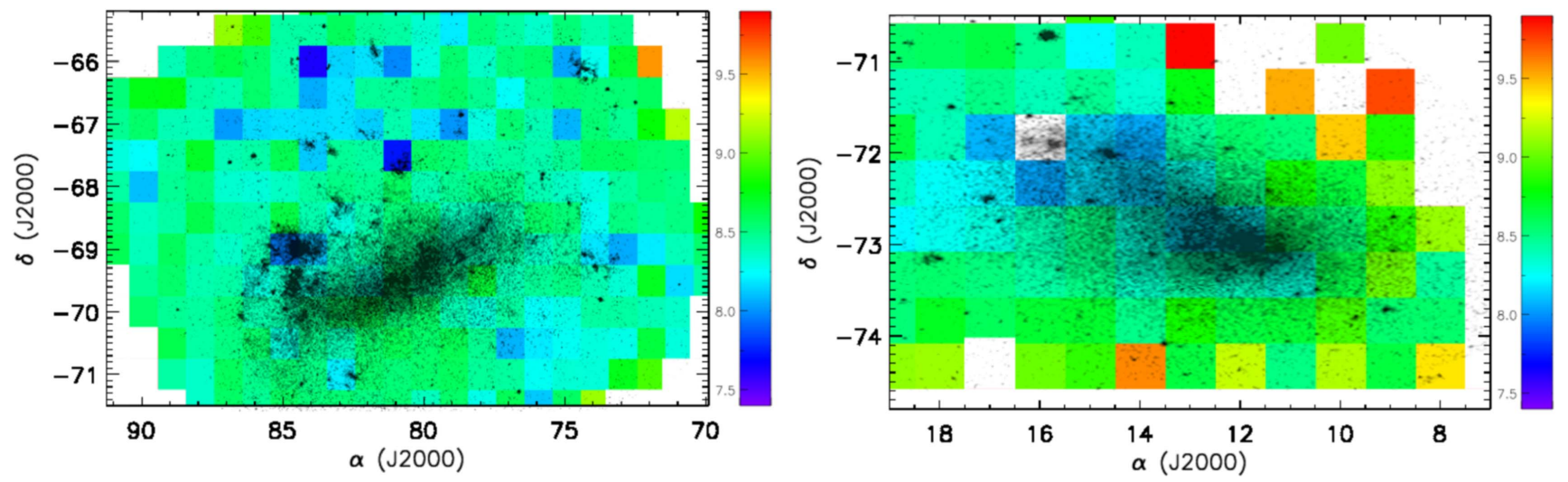

Figure 9. Spatially binned median age distribution of the LMC (left) and the SMC (right), respectively, overlaid on their Spitzer/IRAC $3.6 \mu \mathrm{m}$ mosaics. The

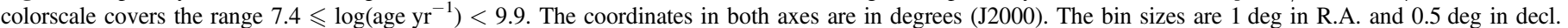

disproven by the absence of a corresponding density enhancement in the old stellar disk as traced by the near-IR, as reported by van der Marel (2001) and confirmed by our own multiwavelength analysis. On the other hand, if the LMC bar was excited or enhanced by an interaction with the SMC a few Myr ago, the present-day star formation in the LMC should still be traced back to that interaction, if indeed star formation is triggered by shocks in the bar-arm interface, especially when the pattern speeds of bar and arms are different (MartínezGarcía \& González-Lópezlira 2011; Beuther et al. 2017).

Our results suggest that, in spite of the asymmetries in the cluster formation histories of the two galaxies, their overall evolution is a combination of both internal and environmental mechanisms. Harris \& Zaritsky $(2004,2009)$ suggested that the star formation histories of the Clouds are dominated by correlated-thus environmental-mechanisms. Our findings agree with their conclusions that the interactions between the Magellanic Clouds and the Galaxy were predominant in uniquely shaping the star cluster formation history in the Clouds.

\section{Conclusions}

We applied our new method to detect and estimate the ages of star clusters in nearby galaxies (originally presented in Bitsakis et al. 2017) on the multiband, high-resolution data of the SMC. We apply the same setup and procedure to analogous data of the two galaxies, and compare the results. Our conclusions are summarized below.

(a) We detect 1319 star clusters in the central $18 \mathrm{deg}^{2}$ of the SMC we surveyed; 1108 of these clusters have never been reported before.

(b) The distribution of cluster ages suggests major star cluster formation $\sim 240 \mathrm{Myr}$ ago. Studying the corresponding distributions of the SMC bar and outskirts, we find that they have significant differences, with the cluster formation peaking at the bar $\sim 200 \mathrm{Myr}$ ago, while for the rest of the galaxy the average age is $\sim 270$ Myr ago. Moreover, the skewness of the age distribution in the galaxy outskirts suggests a termination of the cluster formation over the past few Myr.

(c) The spatially resolved age distribution of the star clusters in the SMC suggests that the inner part of the galaxy was formed more recently, and that an outside-in quenching of cluster formation occurred over the past Gyr. (d) A comparison between the above results and those derived previously for the LMC shows that both galaxies have experienced an intense star cluster formation event at $\sim 300 \mathrm{Myr}$ ago, consistent with a direct collision scenario proposed by model simulations.

(e) Most of the youngest clusters in both Magellanic Clouds are found where their bars meet the H I arms (or similar elongated features), suggesting that cluster formation there is triggered by internal dynamical processes.

(f) Our results suggest that the interactions between Magellanic Clouds are the major driver of their largescale star cluster formation and overall evolution.

The authors wish to thank the anonymous referee for a thorough review and valuable comments that significantly helped improve this article. T.B. would like to acknowledge support from the CONACyT Research Fellowships program. We gratefully acknowledge support from the program for basic research of CONACyT through grant number 252364. G.M. acknowledges support from CONICYT, Programa de Astronomía/PCI, FONDO ALMA 2014, Proyecto No. 31140024. G.B. acknowledges support for this work from UNAM through grant PAPIIT IG100115. This research made use of TOPCAT, an interactive graphical viewer and editor for tabular data. IRAF is distributed by the National Optical Astronomy Observatory, which is operated by the Association of Universities for Research in Astronomy (AURA) under cooperative agreement with the National Science Foundation.

\section{ORCID iDs}

Theodoros Bitsakis (10 https://orcid.org/0000-0001-5787-8242

G. Bruzual (1) https://orcid.org/0000-0002-6971-5755

D. Zaritsky (i) https://orcid.org/0000-0002-5177-727X

S. Charlot (iD https://orcid.org/0000-0003-3458-2275

\section{References}

Baumgardt, H., Parmentier, G., Anders, P., \& Grebel, E. K. 2013, MNRAS, 430, 676

Besla, G., Kallivayalil, N., Hernquist, L., et al. 2007, ApJ, 668, 949

Besla, G., Kallivayalil, N., Hernquist, L., et al. 2012, MNRAS, 421, 2109

Beuther, H., Meidt, S., Schinnerer, E., Paladino, R., \& Leroy, A. 2017, A\&A, 597, A85

Bica, E., Bonatto, C., Dutra, C. M., \& Santos, J. F. C. 2008, MNRAS, 389, 678 Bitsakis, T., Bonfini, P., González-Lópezlira, R. A., et al. 2017, ApJ, 845, 56 Bolatto, A. D., Leroy, A. K., Jameson, K., et al. 2011, ApJ, 741, 12 Boutloukos, S. G., \& Lamers, H. J. G. L. M. 2003, MNRAS, 338, 717 
Chen, Y., Bressan, A., Girardi, L., et al. 2015, MNRAS, 452, 1068

Chiosi, E., Vallenari, A., Held, E. V., Rizzi, L., \& Moretti, A. 2006, A\&A, 452, 179

Cignoni, M., Cole, A. A., Tosi, M., et al. 2013, ApJ, 775, 83

de Grijs, R., \& Goodwin, S. P. 2009, in IAU Symp. 256, The Magellanic System: Stars, Gas, and Galaxies, ed. J. T. Van Loon \& J. M. Oliveira (Cambridge: Cambridge Univ. Press), 311

Dickey, J. M., Mebold, U., Stanimirovic, S., \& Staveley-Smith, L. 2000, ApJ, 536, 756

Fazio, G. G., Hora, J. L., Allen, L. E., et al. 2004, ApJS, 154, 10

Glatt, K., Grebel, E. K., \& Koch, A. 2010, A\&A, 517, A50

Gordon, K. D., Meixner, M., Meade, M. R., et al. 2011, AJ, 142, 102

Harris, J. 2007, ApJ, 658, 345

Harris, J., \& Zaritsky, D. 2004, AJ, 127, 1531

Harris, J., \& Zaritsky, D. 2009, AJ, 138, 1243

Hilditch, R. W., Howarth, I. D., \& Harries, T. J. 2005, MNRAS, 357, 304

Kallivayalil, N., van der Marel, R. P., Besla, G., Anderson, J., \& Alcock, C. 2013, ApJ, 764, 161

Kim, S., Staveley-Smith, L., Dopita, M. A., et al. 2003, ApJS, 148, 473

Lejeune, T., Cuisinier, F., \& Buser, R. 1997, A\&AS, 125, 229

Marigo, P., Bressan, A., Nanni, A., Girardi, L., \& Pumo, M. L. 2013, MNRAS, 434, 488

Martin, D. C., Fanson, J., Schiminovich, D., et al. 2005, ApJL, 619, L1
Martínez-García, E. E., \& González-Lópezlira, R. A. 2011, ApJ, 734, 122

Mighell, K. J., Rich, R. M., Shara, M., \& Fall, S. M. 1996, AJ, 111, 2314

Ochsendorf, B. B., Zinnecker, H., Nayak, O., et al. 2017, NatAs, 1, 268

Olsen, K. A. G., Zaritsky, D., Blum, R. D., Boyer, M. L., \& Gordon, K. D. 2011, ApJ, 737, 29

Parisi, M. C., Geisler, D., Carraro, G., et al. 2014, AJ, 147, 71

Rafelski, M., \& Zaritsky, D. 2005, AJ, 129, 2701

Richardson, S., \& Green, P. J. 1997, Journal of the Royal Statistical Society, 59,731

Rieke, G. H., Young, E. T., Engelbracht, C. W., et al. 2004, ApJS, 154, 25 Schmeja, S. 2011, AN, 332, 172

Siegel, M. H., Porterfield, B. L., Linevsky, J. S., et al. 2014, AJ, 148, 131

Simons, R., Thilker, D., Bianchi, L., \& Wyder, T. 2014, AdSpR, 53, 939

Stanimirovic, S., Staveley-Smith, L., Dickey, J. M., Sault, R. J., \& Snowden, S. L. 1999, MNRAS, 302, 417

Stetson, P. B. 1987, PASP, 99, 191

van der Marel, R. P. 2001, AJ, 122, 1827

Venn, K. A. 1999, ApJ, 518, 405

Yoshizawa, A. M., \& Noguchi, M. 2003, MNRAS, 339, 1135

Zaritsky, D., Harris, J., Thompson, I. B., Grebel, E. K., \& Massey, P. 2002, AJ, 123,855

Zhang, H.-X., Hunter, D. A., Elmegreen, B. G., Gao, Y., \& Schruba, A. 2012, AJ, 143, 47 